\title{
The real challenge of using digital imaging technology on printed textiles
}

\section{Introduction}

The practice of art \& design is altered with the emergence of digital printing techniques and Computer aided Designs (CAD) programs. The concern of this article is to encourage surface pattern designers to utilize digital imaging in broadening their visual and imaginative capacities in creating more complex artistic surface pattern designs for printed textiles.

In the later half of $20^{\text {th }}$ century, technology has advanced to such a level that it affects all other professions, thinkable. What may seem astonishing is the fact that technology has also re-defined aesthetics, art and the very idea of creativity itself. Digital imaging became a handy tool for textile industries internationally. It is not only cost effective but also efficient in meeting the rising demand from the industry. ${ }^{1}$

In earlier times, clothing was a basic necessity which later evolved into fashion. At present, industrialization has altered the economy and enhanced urbanization. Urbanization in turn promoted luxurious living and high tech lifestyle. This led to high demands in textile not only to increase production in the fashion and textile industry but also to produce complex and more artistic surface pattern designs which in the past seemed difficult or next to impossible.

By definition, digital printing is the process of printing digital images directly on to a variety of media substrates. ${ }^{2}$

Digital imaging or digital image acquisition is the creation of digital images, such as of a physical scene or of the interior structure of an object. The term is often assumed to imply or include the processing, compression, storage, printing, and display of such images. ${ }^{2}$

Digital imaging is being used as an effective tool in the printing industry. The benefits of digital printing and imaging are endless. It is cost effective as compared to hand crafting where when you have to experiment on various sampling to attain perfect color coordination and tones. With the aid of specialized software, now designers can modify tones, hue and saturation without undergoing actual sampling. ${ }^{3}$ Multiple layering and various tools helps to create more complex, rhythmic and naturalistic designs possible rather than repeating patterns and motifs. Moreover, digital imaging has opened the doorway of options when it comes to bringing in more depth and meaning in surface pattern design.

Despite numerous benefits of digital imaging, according to Cathy Treadaway, the true potential of digital imaging is not yet realized. ${ }^{4}$ There is hidden knowledge and skills within the domain to play and experiment by designer until he actualizes his or her imagination by collaborating it with technology in producing something interesting and complex.

Yet surface pattern designers attached to fashion and home textile industries are not able to benefit from the digital imaging technology to fullest potential. Many factors may come into consideration when it comes to the artistic use of this wonderful technology as a tool to unleash the language of art. One major area seems to be the non artistic designers using the technology, not knowing the true depth of
Volume 6 Issue 2 - 2020

\author{
Adil Masood Qazi, Humda Khan, Amna \\ Manzoor \\ Department of Design, University of Management and \\ Technology, Pakistan
}

Correspondence: Adil Masood Qazi, Department of Design, University of Management and Technology, C-II Johar Town, Lahore 54770, Pakistan, Email adil.qazi@umt.edu.pk

Received: February 29, 2020 | Published: April 14, 2020

art as a language is the possible barrier that one can observe easily.

The real challenge to exploit the digital imaging technology is to get trained, creative artistic minds to use this tool in a manner which is more like painting a canvas rather than conventional style of playing around with repeats and adjusting motifs and patterns.

In the era where Briggs claims that photography has created new visual language $;{ }^{5}$ students are unable to reconcile their natural imagination and cognitive ideas onto visual space. This can be eliminated by making textile and design students comprehend digital imaging in a better way.

Digital imaging is often criticized for lagging in creativity (since it is computer aided) and lacking in aura. ${ }^{6}$ The overall criticism is initiated by creating a dichotomy between creativity and technology. If instructors could make them understand that digital imaging is visualizing the concepts before they are actualized as an artifact on fabric. Furthermore, creating digital imaging in virtual space is the collaboration of ones shared experience, cognitive capacities, aesthetics, production growth motivation and technology.

Another way to cope with it is by overlooking the positive and negative impact of digital imaging and focusing on neutralizing both impacts by making thought visible through shared imagination. For instance, either digital images have affected creativity the way it was practiced via hands, sketches, vision, touch and cognitive connection [negative impact]. Or it has provided source material for new concept initiations for designers to be explored [positive impact]. Design students fall in either of aforementioned categories: hence the former couldn't feel comfortable with the newly formed techniques and the latter couldn't reconcile creativity with technology or utilize real potential of digital imaging. This can be resolved by neutralizing the two concepts as tools and medium consisting of immense ideas which the designers have to explore, experiment and play in order to achieve desired results.

The world we live in changes at a fast pace with respect to the demands and supply concepts, whereas technology is smartly bridging the two ends. The only real challenge for the surface pattern designer is to make use of the imaging and digital printing technology thinking it to be canvas they bravely paint in interpreting their imaginative ideas. 
The consumer market has always welcomed change, it is only a matter of giving them the option to opt for more artistic and creative surface pattern designs for fashion and home textiles industries. Together technology and art has done wonders in the past, and they will continue doing so in future.

\section{Acknowledgments}

None.

\section{Funding}

None.

\section{Conflicts of interest}

Author declares there is no conflict of interest.

\section{References}

1. Briggs A, Bunce GE. Breaking the Rules: Innovatory uses of CAD in printed textiles. Ars Textrina. 1995;24:185-203.

2. Digital imaging. definitions.net; 2019.

3. Rouse Margaret. What is digital printing. What is; 2010.

4. Treadaway Cathy. Digital creativity: the impact of digital imaging technology on the creative practice of printed textile and surface pattern design. Textile \& Apparel, Technology \& Management. 2004;4(1):1-12.

5. Treadaway Cathy. Digital imagination: The impact of digital imaging on printedmtextiles. Textile: Cloth and Culture. 2004;2(3):2-14.

6. Using empathy to research creativity: collaborative investigations into distributed digital textile art and design practice. 6th Conference on Creativity \& Cognition. Washington; 2007:63-72. 